\title{
Informing Caregivers Through an Assistive Tool: An Investigation of Elderly Care Metrics
}

\author{
Simon Klakegg ${ }^{1}$, Niels van Berkel ${ }^{2}$, Aku Visuri ${ }^{1}$, Chu Luo ${ }^{2}$, \\ Jorge Goncalves ${ }^{2}$, Simo Hosio ${ }^{1}$, Hanna-Leena Huttunen ${ }^{1}$, Denzil Ferreira ${ }^{1}$ \\ simon.klakegg@oulu.fi, n.vanberkel@student.unimelb.edu.au, aku.visuri@oulu.fi, chul3@student.unimelb.edu.au, \\ jorge.goncalves@unimelb.edu.au,simo.hosio@oulu.fi, hanna-leena.huttunen@oulu.fi, denzil.ferreira@oulu.fi \\ ${ }^{1}$ Center for Ubiquitous Computing, University of Oulu \\ ${ }^{2}$ Interaction Design Lab, University of Melbourne
}

\begin{abstract}
Elderly care is a pressing societal challenge: government's financial burden is expected to exponentially increase in the next 20 years as the population is aging rapidly. Solutions to mitigate this challenge include the use of IoT and software solutions to minimise the effort of elderly care, in care centres and at home. To accomplish this, we set to quantify what are the most important elderly care metrics (i.e., what is important to support caregivers' work) through field observations and interviews at a local care centre housing 14 old adults. We designed iteratively and evaluated the usefulness of a mobile application with 8 caregivers, to summarise and communicate the care metrics, juxtaposed with wellbeing data (e.g., social interaction, mobility and others), part of a larger elderly care support platform, CARE. The goal of the mobile application is to enable a better care service by raising awareness to daily needs and routines of the elderly and to provide quick access to their wellbeing information. Our findings advocate that our design could positively benefit the care personnel and assist them carrying out the daily duties at the care centre.
\end{abstract}

Instrumentation, wellbeing, healthcare metrics, applications, prototype, user-centred design

\section{INTRODUCTION}

Globally, society is ageing at a fast pace [12], with the population aged 60 and over representing the fastest-growing group [33]. In the U.S., 46.2 million citizens (14.58\%) are over 65 years (2014), and this number is expected to grow to more than 98 million by 2060 [2]. These demographic changes in society affect family stability, increase caregivers' workload, and consequently lead to a severe upshot on the national health expenses [7]. According to an National Health Expenditure report published in 2015, the U.S healthcare system is facing increasing costs [28], spending approximately $\$ 18,988$ per year, per senior capita, on health care services. In addition, the elderly still represent the smallest population group, but already in 2012 they accounted for approximately $34 \%$ of all expenditures

A direct consequence of the accumulating demand on elderly care services, is a strenuous work environment for caregivers. High workload and stress are key underpinning factors contributing to this outcome [9]. The occupation has endured high turnover rates which has affected the quality of care [32]. In such an environment, the care needs of the elderly may go unnoticed by medical personnel. As old adults experience different kinds of losses (e.g., death of loved ones, impairing illness), social isolation can become a severe threat, leading to major health problems among the elderly [14].

To alleviate caregivers' challenges, new pervasive healthcare systems must be created to support old adults and caregivers. For example, by using sensors, we can identify medicine [17], monitor imperative user data (e.g., biosignals) [21] and user's context (e.g., activity, location) $[4,10]$ and inform caregivers. Technology can improve caregivers' effectiveness [30], enhance the care service [18], reduce operational costs [1] and assist in decision-making [15]. However, such technology is met with low user acceptance [13], due to a lack of focus on the end-users' functional requirements, during the design and implementation phases [21].

To tackle low user acceptance, we present our initial work towards understanding the care needs of the elderly, from within the current care facilities, and how to inform the caregivers on relevant data through a handheld Android tablet application. To quantify the care needs of older adults, we conduct a field study at a local nursing home. With the use of observations and semi-structured interviews, we investigate the daily challenges faced by caregivers in such a workplace context. We created an Androidbased application to aid the caregivers in their daily tasks and help raise awareness to the needs of the old adults. Finally, we evaluate the application 
through a user study. The contribution of our work is three-fold:

- We quantify old adults' care needs through observation and caregiver interviews, and propose a set of old adult care metrics;

- We evaluate how these caring metrics can best be communicated to care workers through a user study at a care centre;

- We evaluate a design of a mobile application to assist caregivers in their daily tasks, aiming to raise awareness of the old adults' needs through the care metrics.

\section{RELATED WORK}

\subsection{Pervasive Healthcare Systems}

Pervasive healthcare plays an increasingly larger role in our modern healthcare systems. The main momentum behind this evolution are growing costs, the avoidance of medical errors, and increased stress levels among health care providers [35]. The rapid development within mobile healthcare devices and accompanying data infrastructure (e.g., Wi-Fi, 5G, 'Cloud') accommodates for new medical concepts and frameworks.

Madeira et al. [21] built a ubiquitous healthcare system by equipping wheelchairs and assistive walkers with sensors. To prevent critical situations, the risk associated with the measured heart rate, heart rate variability and motion signals is displayed to the resident, and remotely to caregivers. It therefore enables elderly to live more independently. Taleb et al. [29] presents a framework aimed at: i) integrating sensors and actuators needed to monitor and guarantee elderly safety, ii) detecting possibly dangerous situations for the elderly, and iii) composing local emergency response groups of volunteers and caregivers, willing to help in case of an emergency event. The system consists of sensors and actuators (e.g., video cameras, RFID readers, sound sensors) and is evaluated through a case study for visually impaired. The results were promising, but they discovered a need for better security and further research. Ren et al. [27] investigates the various aspects of a mobile healthcare system, such as the Quality of Service (QoS) of the resident monitoring and the security and privacy concerns related to the data. These parameters are discussed in the context of a body sensor network (BSN) which can consist of devices such as EEG, ECG, blood pressure, and pulse sensors. The authors then propose multiple techniques to improve the reliability of the data communication (e.g., multicast transmission and broadcast transmission) and to address the security and privacy concerns for this sensitive information (e.g., using electrocardiogram signals to generate cryptographic keys). Chu et al. [20] developed a data hiding method for smartphones, enabling them to embed and encrypt sensitive data (e.g., medical readings) into carrier streams of other sensors.

\subsection{Healthcare Workplace Tools}

Researchers have developed tools to support caregivers, focusing on problems experienced by healthcare staff. Bardram et al. [4] implemented and evaluated a context aware system in a hospital environment. The study revealed that one of the most beneficial parts of the system, was to display and distribute context information - improving the coordination among the workers. Showing the personal context (e.g., location, status, and activity) of hospital staff was accepted and not perceived as a privacy concern. However, there were certain areas in which the staff did not wish to be tracked (e.g., coffee room, toilet). The information was displayed on personal and public displays to support coordination, scheduling, and social awareness. Bluetooth tags and mobile phones were used to infer caregiver context, and quickly replaced the previous context monitoring and paper scheduling system. Kjeldskov et al. [16] implemented a mobile-context aware electronic patient record (EPR) system at a hospital. They then conduct a usability study comparing this solution to the already existing PCbased system. The results indicate that the mobile system can help support work activities in healthcare settings, but that the interaction design is an important component that needs thorough evaluation.

Reflection is an important method to improve work performance. It is common practice for workers in health care to recall previous work events, either individually or in groups, to analyse how they could improve or enhance their current practices based on this experience. Prilla et al. [25] created and evaluated a smart device application for caregivers. The application collects data during work events to support reflection in scenarios such as handover sessions. The result indicates that the application had a positive effect on the reflective collaboration, which is fundamental to improve work practices. The handover is a critical component of caregivers' workday and is defined as: "Transfer of professional responsibility and accountability for some or all aspects of care for a resident, or groups of residents, to another person or professional group on a temporary or permanent basis" [3]. It is regarded as one of the five biggest challenges against resident safety by the WHO [24].

A challenge facing mobile healthcare is the adoption rate of the introduced technology by professionals. It is imperative to understand why caregivers or medical doctors choose to use this technology to support their work, or why they may decline such usage. An extensive study by $\mathrm{Wu}$ et al. [36] 
investigates the primary factors behind medical technology acceptance. They conclude by suggesting that the systems should have a high perceived service availability (PSA), as this is strongly linked with perceived usefulness (PSU) and the attitude of users. They also encourage designers to examine the functional requirements of users, so that the system can provide useful information that helps healthcare professional in making decisions. This would further increase the value of the tool for the users, and make it more likely that they use it.

\subsection{Mobile Wellbeing}

Tollmar et al. [31] created a system in which a range of data is gathered through multiple sensors, i.e., Withings (weight and body fat), Fitbit (step count, sleep and awake hour), Android Calendar (busy time), Experience Sampling Method (ESM) [8] (location, exercise and food). Data is collected during the day and statistical relationships regarding someone's wellbeing is calculated during the night. The results are presented to the users in form of a small Android widget, such as: "On Fridays you walk less than on Mondays", or "On busy days you eat more." This allows users to notice how their wellbeing trends changes with their context, and learn new information about their living habits. Eighty percent of the participants in the study lost weight and report an increase in the WHO-5 WellBeing Index. BeWell [19] is an application that utilises the smartphone sensors physical, social, and sleep dimensions in a wellbeing context, monitors behaviour, and provides feedback to the user. The purpose of this feedback is to inform the user on their behaviour, so they may understand the consequence of their choices and enabling them to make informed (health related) decisions going forward. Users displayed a significant diversity, with no golden standard for wellbeing patterns for all (i.e., it is challenging to generalise health metrics, users must often be considered individually). The results indicate a positive trend in behaviour change, but a longer study is needed to strictly verify such findings.

Rashidi et al. [26] conducted an extensive survey on living technologies based on ambient intelligence (i.e., technology which aim is to empower humans' skills, while being sensitive, flexible, and responsive to our needs). Technology implementing these principles to assist people in daily life and increase their wellbeing, are called ambient-assisted living $(A A L)$ tools. They conclude by stating that these systems create new opportunities for innovative healthcare system due to the technological development of mobile and wearable sensors.

\subsection{Positioning our work}

Our work contributes towards a better understanding of the care needs of the elderly, from within the current care facilities and practices. Our designed Android-based application aims to support the caregivers in their daily tasks and raise awareness to the needs of the old adults and facilitate collaboration in the caregivers' team. We quantify old adults' care needs (i.e., care metrics) and evaluate their importance and how they can be presented and accessed by the caregivers on-thego without disrupting their daily tasks. The end result is a tool that is built from the ground-up with the caregivers, instead of a solution that is enforced upon the caregivers' existing work practices.

\section{FIELD STUDY: OBSERVATIONS, SCHEDULES, TECHNOLOGY}

To create a solution to assist elderly caregivers, we first set to investigate and better understand their core work needs in operating within a nursing home. 4 researchers scheduled a 3-day long field study to a local nursing home. We conducted semistructured interviews with the care facilities manager and 8 caregivers, and observed the caregivers' daily routines and interactions with the residents. We focused on identifying potential workflow breakdowns (i.e., time consuming or progress halting) that could be mitigated with mobile and sensing technologies, i.e., by automation of wellbeing assessment of a resident; new communication channels between stakeholders (e.g., caregivers, manager, family members).

\subsection{Old Adults' Home}

The care centre measures approximately $440 \mathrm{~m}^{2}$, and consists of 14 resident rooms, two hallways, a public lounge, and a kitchen area. The section of the nursing home we visited currently houses 14 residents. The majority of residents are old adults (i.e., 65 years or older) with delimiting health (e.g., Alzheimer's, dementia, memory loss). Therefore, the care centre strives to replicate a home and familiar environment (e.g., there are family pictures in residents' homes, familiar furniture items), and the residents can walk around the facility without restrictions during the daytime. Besides the regular caregivers, there are also cleaning personnel and care students present in the facilities. The students are there as part of their work practice and preparation, and are the main source of renewed working staff. The students typically have no prior knowledge of the residents' needs and their background, and are trained on-site.

\subsection{Work Schedule}

The care schedule currently operates in three shifts: morning (07:00 - 15:00), day (13:00 - 21:00), and night $(21: 00-07: 00)$. From 13:00 to $15: 00$, there is an overlap between the two shifts and the caregivers have a meeting (i.e., the handover) where they discuss the residents' status during the past day. 
There are two caregivers present during the morning and day shifts, and only one working during nights and weekends. Nights and weekends were unanimously regarded as highly stressful shifts. In addition, certain events pose extra challenges to the staff than others (e.g., mealtimes are particularly busy as many residents need help concurrently).

During the field study, we observed the residents to follow a relatively flexible daily schedule, with occasional events with a fixed schedule (e.g., breakfast 07:00, lunch 11:00, dinner 17:00, supper 19:00, bedtime 22:00). There are additional events which do not follow a rigorous schedule, such as casual resident check-ups, room cleaning, group exercise and social activities, dependent of the day and seasonal availability. Lastly, there are sporadic events (or, incidents) which occur at random (e.g., resident falls and needs assistance, resident needs medical attention).

\subsection{Technology}

Pressure mats are placed beside the beds, if there is a reasonable doubt that the resident is prone to fall from the bed. The mats are connected to a speaker that notifies the caregivers when pressure is applied, indicating that the resident is either standing up, or may have fallen from the bed. The caregivers always carry a speakerphone which is connected to alarm bands given to a selected set of residents. By pressing a button in the band, the residents can notify the caregivers that they are in need of assistance.

The speakerphones do not allow for direct communication, but simply voice an alarm. Lastly, the facilities possess a computer-based logging system, used in a centralized fashion for reporting and retrieving resident information, i.e., caregivers need to move towards a specific room (Figure 1) and computer to log and retrieve information, majority of it on paper in a file or hanging on a wall.

\section{CARE METRICS}

Using affinity diagrams, concept aggregation and note matching, we identify 18 distinct events, concepts or items which we name care metrics.

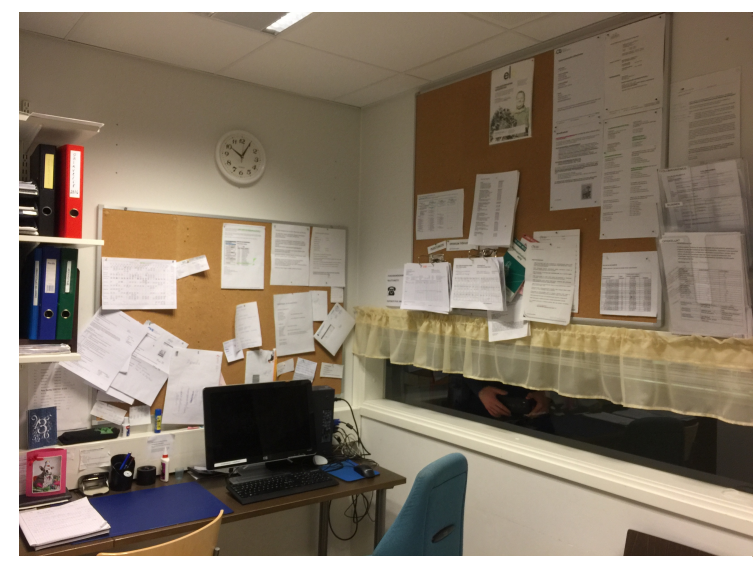

Figure 1: Nurses' office with computer to log information and paper based information sources on the wall

For us, a care metric is a unit of information that may be measured and observed as part of the elderly care work practices. The metrics are autonomous information sources that continuously change throughout the day. These metrics can be collected either automatically using sensors, or through manual input by the caregivers (e.g., using ESM). Table 1 lists the resulting potential care metrics, along with an icon we selected to communicate the metric in our application.

\subsection{Validation}

To validate the care metrics, we scheduled a new visit at the care centre. We interviewed 8 caregivers ( 2 males, 6 females, age 22 to 35 years, SD $=5.39$ ), with work experience ranging from 1 to 15 years $(M=10.13, S D=5.82)$, to rate the importance of each individual metric in relation to their work on a 5-point Likert scale. Since icon interpretation can be subjective, we shortly described the context of each care metric, so that each participant would have an understanding of its meaning. The ranking by mean value (highest first), are shown in Table 2 . There is a considerable difference in the perceived usefulness and importance of the presented care metrics. Sleep is deemed most important, as it gives a good indication of the resident's wellbeing and is challenging to check upon without disturbing during the night.

Table 1: Overview of visual representation of information segments.

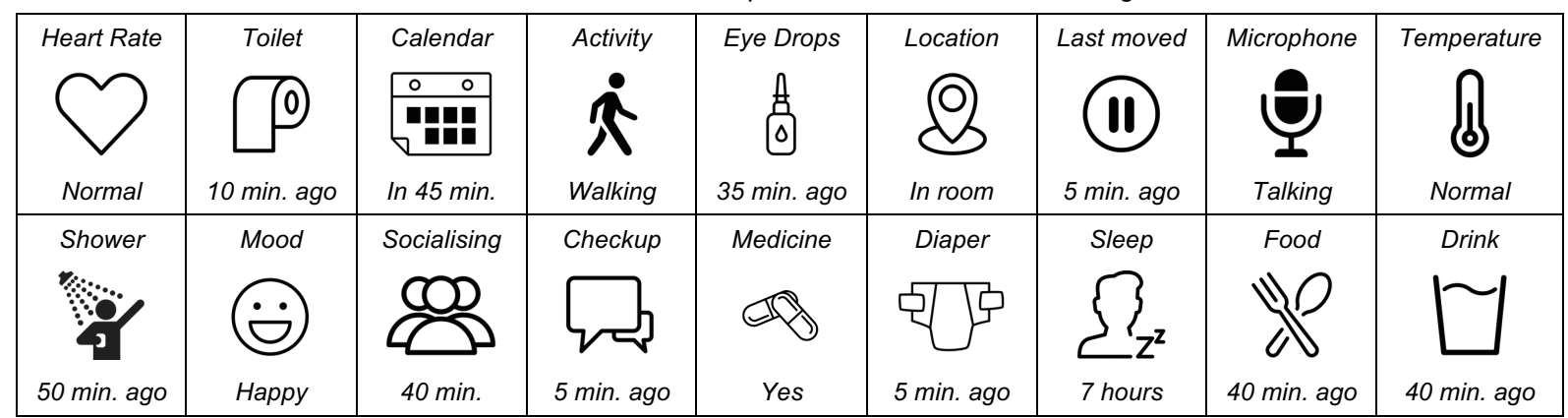


Informing Caregivers Through an Assistive Tool: An Investigation of Elderly Care Metrics Klakegg • van Berkel • Visuri • Luo • Goncalves • Hosio • Huttunen • Ferreira

Table 2: Importance of potential care metrics ranked on a five-point Likert scale.

\begin{tabular}{|l|c|c|}
\hline \multicolumn{1}{|c|}{ Care Metric } & Mean & SD \\
\hline Sleep & 4.75 & 0.46 \\
\hline Activity & 4.62 & 0.74 \\
\hline Toilet & 4.50 & 0.53 \\
\hline Eye Drops & 4.37 & 0.91 \\
\hline Location & 4.28 & 0.75 \\
\hline Food & 4.00 & 0.92 \\
\hline Drink & 4.00 & 0.92 \\
\hline Temperature & 3.87 & 1.12 \\
\hline Heart Rate & 3.75 & 1.03 \\
\hline Check-up & 3.62 & 1.59 \\
\hline Microphone & 3.37 & 1.50 \\
\hline Last Moved & 3.37 & 0.74 \\
\hline Medicine & 3.37 & 1.59 \\
\hline Diaper & 3.25 & 1.90 \\
\hline Socialising & 3.00 & 1.41 \\
\hline Calendar & 2.87 & 1.45 \\
\hline Shower & 2.62 & 1.59 \\
\hline Mood & 2.37 & 1.30 \\
\hline
\end{tabular}

Equally important are eating, drinking, and toilet metrics. If, and when the health of a resident deteriorates, it tends to impact these metrics first.

It is also important to know when certain residents (e.g., mobility impaired, bedridden) move, which is reflected by the activity score. Multiple resident miscellaneous needs (e.g., toilet, eye drops, drink water, check-up) could sometimes be skipped by staff, and they would appreciate a reminder. Measurement of bio-signals (e.g., body temperature, heart rate) was considered unsuitable for a care centre context (i.e., no wearables policy), but still received above average scores due to their importance. Metrics such as socialising and mood were not considered very interesting to the caregivers, perceived as being too random (e.g., social opportunities are frequent and event-driven, and mood of a resident fluctuates frequently during a day). However, a longitudinal indication of the social metrics would potentially be of interest, as they may reveal trends such as social isolation, depression. Lastly, shower received a low score as it is predetermined within the existing care schedule.

\subsection{Caregiver Assistant: Prototype}

We presented high fidelity sketches of a handheld Android-based application (Figure 2) to the same nurses who participated in the care metric validation. The application consists of four main screens, which we describe next. The overview screen (Figure 2 left) provides a list of the residents in the care centre, and the most important care metrics, modifiable by the caregivers. Within a row, the order of each metric is dependent on the time elapsed since the last event (e.g., five hours since last toilet visit takes precedence over displaying hours of sleep). Tapping an icon has no action. By selecting a resident on the left side of the list, caregivers are presented with the resident's medical information (Figure 2 - middle). The application also allows caregivers to show information on the resident's family and directly contact them if necessary (Figure 2 - right). Lastly, the application offers a summary of the resident's personality and describes his likes and dislikes (screen not shown). We present this concept application as a tool to assist the caregivers during their workdays, by informing them on the elderly
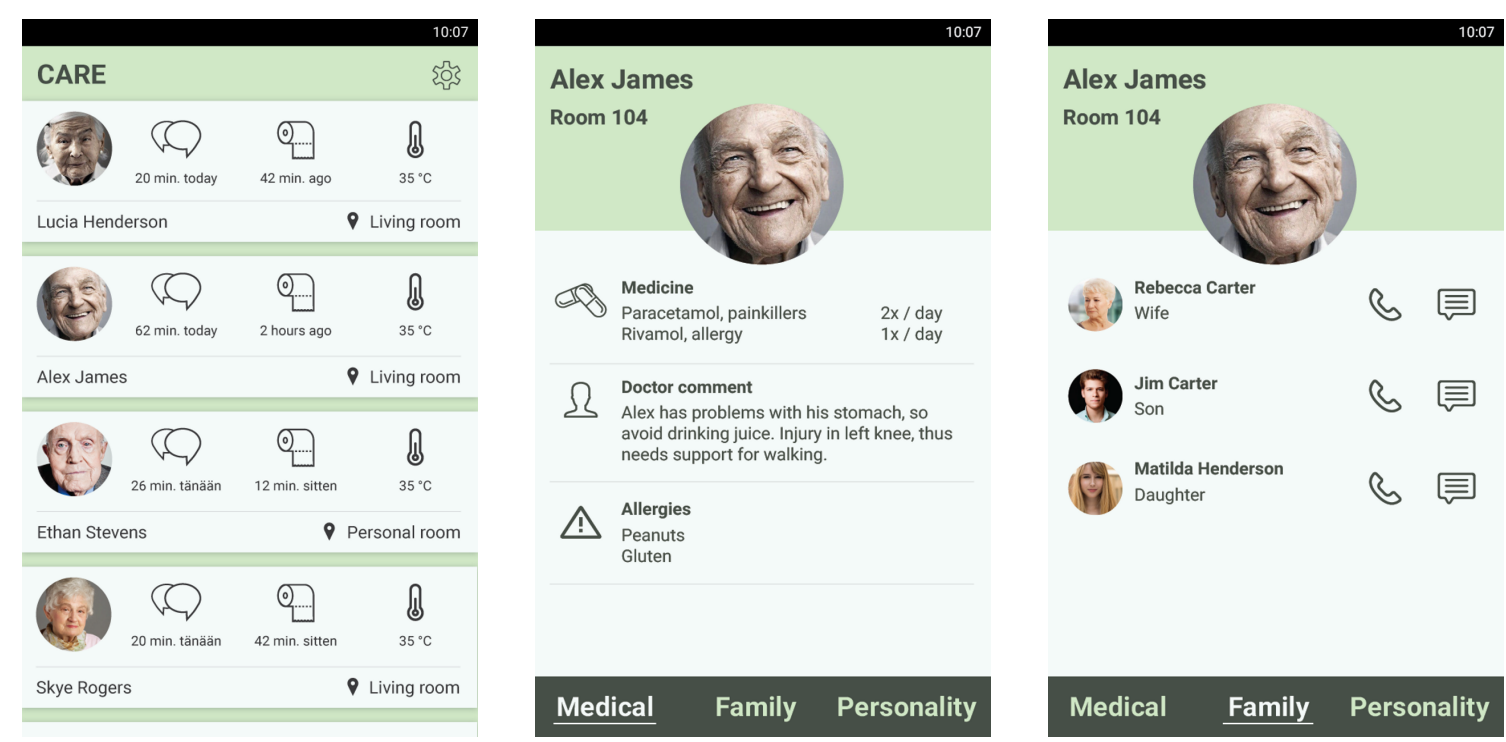

Figure 2: High fidelity sketches of the UI. 
care needs and relevant information, and allowing them to coordinate.

To evaluate our application, we used the think-aloud protocol [11] in combination with a semi-structured interview. A think-aloud evaluation enables participants to verbalise their thoughts as they navigate on the application. We presented the various UI sketches to 8 available caregivers (5 female, 3 male) aged between 17 and 35 (SD = 5.66), with work experience ranging from 1 to 17 years $(M=6.38, S D=6.16)$, and asked them for feedback on aspects of usability, usefulness, importance and presentation of the information, and general perceived usefulness of the application. At this stage of the evaluation, we were interested in the caregivers' collective feedback of the prototype application as an assistive tool in their daily work and not the technical details nor the accuracy of the measurements. We aimed to investigate how the application fits into their daily tasks and work practices, and which aspects (e.g., functionality, services) of the application they did or did not like.

\subsubsection{Utility}

The caregivers emphasised that although a lot of the resident background information is available in the PC based system, it is too time consuming to retrieve it when they want to access it. They would like to be able to access this information without having to leave the care room. This is also extremely important for emergencies, as retrieving instructions or warnings such as DNR - a requirement which only came apparent to us after the evaluation - is time-critical.

Multiple comments were made about the utility of our application, compared to the current PC based system: "It is a great tool to help memorise things. The current system is very heavy and cumbersome to use" (P01). "It is a faster system than current one, it seems simple to use and information is easily readable" (P07). "It is a bit hard to get an overview of how the system would work. But it has a lot of potential because it's portable and can start up fast." (P01). "It would be helpful because it saves us time - there is no need to run back and forth. We always need to go back and check information, either on paper or with the PC-based system (really slow), when asked about it to make sure it's verified" (P05). "It is also really useful during night shifts" (P01). We must note that the aim of our application is not to replace the existing $P C$ based resident management system. Rather, the goal is to provide caregivers with information used in their daily work - which exist on the PC based system - in a more convenient way, in order to minimise their decision-making time and therefore improve the quality of care.

\subsubsection{Usefulness}

The interviewed caregivers also commended on the usefulness of the information in the medical, family and personality layouts, and how it suits the mobile format. "It helps part-time workers and students immensely, as the app might reduce mistakes they make in their daily work and thus makes it less likely for the customers to get upset over these mistakes. Around one third of all employees are rotating employees (e.g., summer interns, replacement workers due to maternity leave or sick leave, students in their practice period)" (P02). "It can take weeks or months for new caregivers to learn to know all the customers without any aids" (P08). "Great tool for introducing new caregivers, and also helps old caregivers by reminding them" (P07).

\subsubsection{Residents wellbeing}

P6 underlined how this tool also indirectly benefits the residents. "Logging increases the wellbeing of the customers - sometimes staff assumes a thing has been done, especially if a resident is the type who wanders around or is very independent, but in truth nobody has performed the task for a while" (P06).

\subsubsection{Situational awareness}

The caregivers stated how this could also aid them with an up-to-date overview of the old adults without inconvenience. "It is a good idea, no need to check on the residents constantly" (P03). "The overview has sufficient information presented of each customer" (P02). "The extra work that we are required to do when logging the information is not too much" (P07, P08).

\subsubsection{Presentation}

Finally, the participants seemed satisfied with the visual presentation of the information. "Looks good, it's clear what is presented" (P07). "It is easy to recognise people by using both picture and name. There is not too much text" (P08). "It is clearer to new people, so attaching a name to a face is very helpful" (P03).

\section{CAREGIVER ASSISTANT: FINAL DESIGN}

Our main goal is to present relevant information to the caregivers concerning the residents' wellbeing, background, and care needs. This enables caregivers to make informed decisions about which tasks to perform next, thus potentially increasing work efficiency. It is also meant to assist new workers (e.g., students, rotating staff) to quickly familiarise themselves with all the residents.

\subsection{Design}

While we consider it out of scope for this paper to describe the technical workings of this system in detail, it is designed to be unobtrusive and support the workflow of care personnel without enforcing additional labour on the user. The sensor infrastructure which provides automated contextual 
information about residents consists of an array of loT devices (ThingseePOD $\left.{ }^{1}\right)$, BLE beacons $\left(\right.$ Estimote $^{2}$ ), Android PCs (Remix Mini ${ }^{3}$ ), and a Raspberry Pi 3 for collecting the data and uploading it to the cloud for further processing, data storage, and enable other applications. The technical evaluation of CARE infrastructure is ongoing and will be published at a later stage.

Thus, the application presented here acts as the interface between the data collected by the sensor infrastructure and the end-user, i.e. caregiver. We designed the UI based on Nielsen's usability heuristics [23], establishing an application that can be quickly understood by its users. For example, we use their terminology to create a match between the system and the real world. In addition, the application highlights critical patient needs to minimise the cognitive load, enabling recognition rather than recall. Furthermore, we follow Android guidelines for Material Design [22] to present users with a familiar design and interaction paradigm. Figure 3 displays some of the fundamental final layouts in our application.

The main overview (Figure 3 - left) contains a list of all the residents in the care centre. For each resident, their name and pictures is displayed, followed by a set of care metrics to the right. The list of care metrics aims to give the caregivers an overview of the current situation in the care centre, and assist them in making decision about what task to complete next. The metrics are sorted from left to right (with a maximum of four needs) based upon urgency and are updated automatically as new data is collected. A care metric in need of imminent
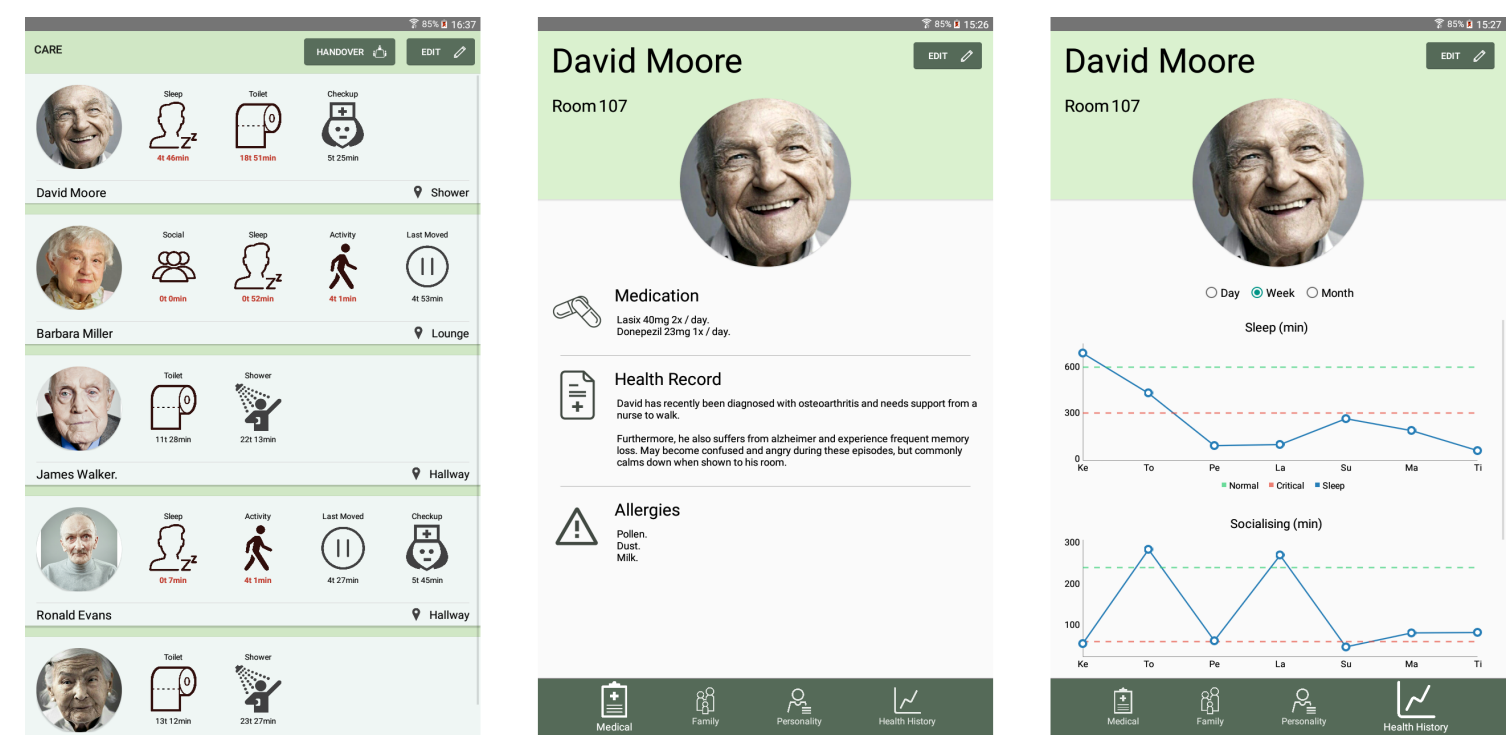

Figure 3: Final design of the implemented CARE Assistant

attention or consideration has its text displayed in red (i.e., resident only slept 2 hours the previous night). The bottom right corner of each card shows the current location of the resident to enable the staff to easily locate a resident if needed. When the edit button is pressed, the user can add new residents or remove existing ones. The main overview presents large icons and little text, so users can efficiently retrieve information on the current context at a glance.

When the user requires more in-depth information they can open the resident detail view (Figure 3 middle) by tapping on a resident. Resident detail view contains four tabs (medical, family, personality, and health history). For each tab, there is an edit button that allows the user to edit the information (e.g., name, room, picture, information). The medical view contains information about the medication the resident should take, a health record, and a list of allergies. This is the information presented first, as the caregivers described medical information as most important. The family tab enables care personnel to have an overview of relatives by presenting a list of close family members with name, photo, number, and relationship description. Additionally, it is possible to remove and add new family members. There is a call and text button next to each person to accommodate effortless and quick communication. Information on the resident's background, hobbies, likes, or dislikes, can be found in the personality tab.

The health history tab (Figure 3 - right) displays graphs of the resident's wellbeing (e.g., sleep, activity, and socialising patterns). All graphs contain
${ }^{1}$ https://www.haltian.com/Internet-Of-Things/Thingsee$P O D$-and-TAG 
a green dashed line, indicating a healthy minimumthreshold, where everything beyond this line is considered healthy and common. Furthermore, the graphs also incorporate a red line indicating the critical threshold, where any values beyond this are a reason of concern for the caregivers. Using this historical overview, caregivers can assess the health development of residents over extended periods of time, with the possibility to switch between day, week, and month viewing mode. This enables the caregivers to observe changes in the resident's health, which may not be visible on a day-to-day basis, but can be very significant and revealing over a longer period. Ultimately, the overview can be used for reflection and analysis on the resident's status.

Finally, the main overview enables access to the handover mode. This mode is specifically designed to guide the caregivers during their handover meetings. Upon activating the handover mode, the application shows a resident's health history - like the health history tab - and three 5-point Likert scales (mood, appetite, and health). These scales allow the caregivers to quickly add data on wellbeing metrics that can be challenging to automatically monitor using sensors. Longitudinal fluctuation of these metrics, in combination with sensor data, can then be used by the caregivers to assess the resident's care needs during the handover meeting. The information also aims to facilitate reflection upon the resident's wellbeing and health changes. By pressing next, the next resident is presented until all residents have been discussed. By utilising the application to form the basis of the handover meeting, the caregivers can be less prone to lapses (e.g., forgetting to discuss a specific resident or overlooking information).

\subsection{User Study}

To evaluate our application, we conducted a user study with 8 caregivers (2 males, 6 females, age 17 to 35 years $(S D=5.95)$, with work experience ranging from 0 to 17 years $(M=4, S D=6.55)$ at the collaborating care centre. Half of them had also participated in our initial study. First, we briefed the participants about the project and described the purpose of the application. We then used the ThinkAloud protocol [11] and asked the user to navigate through the application, interpret the resident related information presented to them, and try the handover functionality. This allowed us to collect invaluable verbal data from the user's point of view while testing the app. Throughout the study we queried the users with follow-up questions to gain a more in-depth understanding of their thoughts and opinions. In addition, we requested the participants to complete a set of specific tasks that contain the most essential functionalities:
- Add a new resident with name and profile picture.

- Add a new family member with name and profile picture.

- Edit a personality field on one of the residents.

- Inspect a resident's health history and interpret the data.

Finally, the users rated our application based on the System Usability Scale (SUS) [5].

\subsubsection{Information presentation}

P03 comments that new employees often need to ask the more senior colleagues about resident related information and could not pinpoint potentially missing information. In general, the caregivers seemed pleased with the simplicity of the information presented. "Looks nice and clean" (P04). One of the participants suggested us to introduce a hierarchy in the presented family members: "The primary family member should be first on the list and it should also be specified" (P02). Besides, P08 highlighted that DNR information was missing. The caregivers also expressed their opinion on the functionality of the application, such as the health history. "Showing historical data is good in cases where caregivers have been on a vacation, or worked elsewhere for a period. It gives them insight on what has been going on lately" (P01), "The historical view is valuable and the threshold gives a good indication of a resident's status" (P07). Caregivers stated that this information would potentially change their decision-making behaviour. "If a resident is behaving abnormally it would be helpful to have information about potential causes (e.g., poor sleep), right now there is a lack of knowledge that could help us make better decision" (P04). In general, the feedback was highly positive. "It is really good. All the information needed is in the app" (P01).

\subsubsection{Navigation}

When the participants navigated the application we noticed some flaws and room for improvement. P01 commented on the back button being slow, and P05 erroneously tried to double tap it because of the delayed response -- resulting in an unintended navigation transition. Both P02 and P04 tried to click the individual sensor icons for more information, which did not present any information. P05 tried clicking the medical tab while being in that specific layout, despite the UI indicating which tab is currently open. Even with these small errors, participants had no problems navigating the application and interacting with the various elements.

The caregivers also commented that the wellbeing icons should more clearly indicate when they pass the critical threshold, either through notifications or more significant visual changes (P07, P08). In addition, the residents could be ordered based on 
their care needs with the most urgent (i.e., residents with critical care metrics) always on top (P06). All participants managed to complete the presented tasks with ease. There were a few minor issues (e.g., occasionally unresponsive buttons, selecting text was difficult as the interactive area was too small, finding family layout from main layout, changing information without pressing the edit button first). Analysis of the SUS revealed a score of 89.4 on a scale from 0 to 100 , which indicates that the participants were satisfied with the application, and that it operated well in terms of both usability and learnability.

\subsubsection{Impact on work}

The handover functionality was described as useful, with one of the caregivers stating that "it reduces misinformation, l'm less likely to forget information" (P04). Both P05 and P06 stated that it is important to also have the opportunity to write notes about the resident, to enable them to describe more in detail, especially in aberrant situations.

Furthermore, the caregivers were positive about the live information updates; "You can observe resident activity from a distance instead of needing to be in direct contact with them" (P02). In addition, it would be easy to prioritise tasks (P03). Concretely, P05 stated that if she noticed a resident has slept poorly, she would let them sleep in and not intrude. There is no need to bother the resident, and caregiver can prioritise to attend to others. This can actually improve the care service as every resident gets attention (P06). "I can see myself using this application, it is helpful because it gives a clear overview of what is going on" (P08).

Some reluctance was also observed; "Difficult to say without actually implementing it and knowing how accurate data each sensor provides" (P04). "It helps clarify information (e.g., reduces pieces of paper and scribbles), it is great for viewing historical data and to share information between colleagues" (P05). Some of the caregivers found the application to be more useful in certain contexts; "It is most useful if you are being really busy and just need to get quick overview of what needs to be done, and the condition of each resident" (P08). "It is beneficial for students and new employees, it helps them learn about residents and identify them and family members" (P04, P05). "I would use the application during breaks" (P03). Obviously the validity and format of information provided by the sensors needs to be validated and evaluated before the final implementation.

\section{DISCUSSION}

Wellbeing is a vital aspect of elderly care and is directly linked to old adults' health status. Furthermore, insufficient social relationships and isolation can lead to severe medical issues [34]. We aimed to empower the caregivers of a care centre with data regarding the resident's wellbeing, to enable them to act on the information and adjust the care to address potential issues. Throughout our discussion and interviews with the care centre personnel, we received positive feedback about how our app would enhance their work environment. We argue that our app can empower caregivers' decision making and make them more aware of residents' needs.

\subsection{Assistive Tools in the Care Centre}

There is a scarcity of research that highlights how technology can support and enhance caregivers working capabilities in-situ, specifically at a care centre. Caregivers currently must conduct a wide array of tasks (e.g., check if resident is in bed, check if resident needs to visit the toilet) that could be made simpler with sensors. Additionally, we argue that there is a need for appropriate tools that can inform caregivers on residents' current care status, allowing them to better plan their work day. In this paper, we identify and evaluate a set of care metrics that can describe the current care needs of residents in a care centre. We then design and evaluate an application that aims to raise awareness of the old adults' needs, by successfully communicating these metrics to caregivers and inform them on other resident related data.

Through our study we identified a lack of tools to assist in long-term care planning and the handover meeting. While a PC based logging system was in place, it was rarely used because of several inconveniences (e.g., complex, slow, located in a separate room). Consequently, caregivers are forced to keep a lot of important resident related information in their memory and share it with coworkers in a verbal and informal manner. This practice is subjective to human error and we aimed to build an application that could mitigate these issues. For example, lack of sleep for a resident may be the source of multiple issues (low appetite, bad mood, sedimentary activity). However, if the caregiver working the night shift forgets to share this information with co-workers, it may lead to faulty decision making. While the handover is common procedure to discuss such knowledge, the strenuous work environment in a care centre can affect the workers cognitive load [6], resulting in loss of important information.

We also examined how components of related systems would integrate in an old adult care centre. For instance, Bardram et al. [4] installed a system which tracked the location and context of workers in a hospital and found a positive effect on worker coordination. We explored the need for a location tracking system (of either caregivers or residents) through our preliminary study. The caregivers 
indicated that it could be beneficial to see the location of residents to get an overview, especially during night time (only one caregiver present). However, they were usually aware of each other's position during the workday, as the premises are fairly small. The caregivers were enthusiastic about having access to resident data on the go, which is in line with a previous study [16] on mobile EPR in a hospital. As another example, previous work $[21,27,29]$ has focused on the medical aspect of care (e.g., measuring heart rate, temperature) and using camera based systems to monitoring old adults. However, such systems were deemed unsuitable by the caregivers as they strive to maintain a home-like setting in the care centre.

\subsection{Enhancing the Elderly Care Service}

The effect of a rising demand on the healthcare systems is more medical errors, costs and stress on providers [35]. To allay these concerns there has been a shift towards the implementation of healthcare technology. While the underpinning infrastructure for integration into elderly care already is in place (e.g., WiFi, 5G, cloud, hardware), challenges still remain for successful integration in a care centre. A key obstacle for successful implementation of a healthcare system is the acceptance rate among medical personnel. In our work we focus strongly on utilising user-centered design, to address the functional requirements of the care staff [36]. Through in-depth observations and interviews, we strived to develop a tool that was suitable for an elderly care workspace.

As a result, our design aims to facilitate care planning and analysis, by monitoring their status and displaying historical data for each resident. This may reveal trends in the residents' health, behaviour and care outcomes. We received affirmative feedback for the handover support our app offers. Its purpose is to inform caregivers on important resident data and ensure that all residents are discussed and analysed during the meeting. Overall, the caregivers believed the application could impact their work in a positive way. The mobile form factor was highlighted as a major benefit, as it always can be kept in close proximity. We also wanted to address the concern of a high influx of personnel (e.g., rotating, new, students), often in need of guidance. The participants commended the applications ability to provide background information about a resident, and recent health history for updating rotating staff. Additionally, the caregivers commented that the application can improve information tracking and it could reduce errors and allowed them to prioritise tasks better through the context overview.

Our application provides a simplified interaction point for caregivers to understand complex data collected from a sensor infrastructure. The simple layouts with large icons and text were designed to help users quickly infer the overall context in the care centre. Our user study shows that participants were able to promptly start using the application reliably, without any further instructions. This is also supported by our application's SUS score (89.375). A number of this magnitude is in the higher end of scores (top 10\%). Applications in this tier is more likely to be recommended to other colleagues, which is very essential for user acceptance among care personnel. For the most part, participants could complete the tasks with minimal effort, however we identified a few minor issues and noted the caregivers' suggestions for improvement. Overall, the caregivers seemed positive about our pervasive sensing approach, and how our application focused on the care needs of the elderly. Such a solution could potentially further extend their working capabilities (i.e., increase efficiency, precision) and naturally be incorporated as a part of their workflow.

\section{CONCLUSION}

In this paper, we present a quantification of elderly care metrics ensuing field observations and caregiver interviews. Following this analysis of elderly care needs, we design and develop a novel application exploiting ubiquitous computing technologies to perform real-time measurements and present the identified care needs in an accessible way to the caregivers. The need for this application follows from the daily work environment consisting of high-pressure decision-making in a high-risk working environment. Our application provides caregivers with both a dynamic overview of care needs, as well as a static overview of resident information -- providing caregivers with a tool to prioritise tasks and access relevant resident information when required. We evaluate our design through interviews, SUS, and a task-based, thinkaloud user study and find a positive reception among caregivers. Our results show a positive evaluation of the introduced concept - citing both expected timesavings and improved quality of care. In future work we will implement the ubiquitous sensing system in our partner care centre and conduct a longitudinal evaluation of our system. Additionally, we will be able to collect valuable sensor data from both patients and nurses in an elderly care centre, which is currently a scarcity. Through in-depth analysis of this data it is possible to derive useful information, to ultimately provide a better care service.

\section{ACKNOWLEDGEMENTS}

This work is partially funded by the Academy of Finland (Grants 276786-AWARE, 286386-CPDSS, 285459-iSCIENCE, 304925-CARE), the European Commission (Grant 6AIKA-A71143-AKAI), and Marie Skłodowska-Curie Actions (645706-GRAGE) 


\section{REFERENCES}

1. Margrethe Aanesen, Ann T. Lotherington and Frank Olsen. 2011. Smarter elder care? A costeffectiveness analysis of implementing technology in elder care. Health Informatics Journal 17, 3: 161-172.

2. Aging Statistics. Retrieved 07/03/2017 from https://aoa.acl.gov/Aging_Statistics/Index.aspx

3. British Medical Association. 2004. Safe handover: safe patients. Guidance on clinical handover for clinicians and managers. London: BMA 7: 141.

4. Jakob E. Bardram, Thomas R. Hansen, Martin Mogensen and Mads Soegaard. 2006. Experiences from real-world deployment of context-aware technologies in a hospital environment. In International Conference on Ubiquitous Computing, 369-386.

5. J. Brooke. 1986. SUS: A Quick-and-Dirty Usability Scale. Usability evaluation in industry. Reading, UK.

6. Paul Cornell, Donna Herrin-Griffith, Courtney Keim, Sarah Petschonek, Adriane M. Sanders, Sidney D'mello, Teresa W. Golden and Gayle Shepherd. 2010. Transforming nursing workflow, part 1: the chaotic nature of nurse activities. Journal of Nursing Administration 40, 9: 366-373.

7. National Research Council and Committee On Population. 2001. Preparing for an aging world: The case for cross-national research. National Academies Press.

8. Mihaly Csikszentmihalyi and Reed Larson. 2014. Validity and Reliability of the Experiencesampling Method. Flow and the foundations of positive psychology. Springer.

9. Maria Engström, Brigitta Ljunggren, Ragny Lindqvist and Marianne Carlsson. 2006. Staff satisfaction with work, perceived quality of care and stress in elderly care: psychometric assessments and associations. Journal of Nursing Management 14, 4: 318-328.

10. Denzil Ferreira, Vassilis Kostakos and Anind K. Dey. 2015. AWARE: mobile context instrumentation framework. Frontiers in ICT 2, 6 : 1-9. http://dx.doi.org/10.3389/fict.2015.00006.

11. Marsha E. Fonteyn, Benjamin Kuipers and Susan J. Grobe. 1993. A description of think aloud method and protocol analysis. Qualitative Health Research 3, 4: 430-441.

12. HelpAge International. Retrieved 02/10/2014 from http://www.helpage.org/

13. Richard J. Holden and Ben-Tzion Karsh. 2010 The technology acceptance model: its past and its future in health care. Journal of biomedical informatics 43, 1: 159-172.

14. Julianne Holt-Lunstad, Timothy B. Smith and J. B. Layton. 2010. Social Relationships and Mortality Risk: A Meta-analytic Review. PLOS Med 7, 7: e1000316. http://dx.doi.org/10.1371/journal.pmed.1000316

15. Simo Hosio, Jorge Goncalves, Niels van Berkel and Simon Klakegg. 2016. Crowdsourcing Situated \& Subjective Knowledge for Decision Support. In International Joint Conference on Pervasive and Ubiquitous Computing Adjunct, ACM, 1478-1483. http://dx.doi.org/10.1145/2968219.2968591.

16. Jesper Kjeldskov and Mikael B. Skov. 2004. Supporting work activities in healthcare by mobile electronic patient records. In Asia-Pacific Conference on Computer Human Interaction, 191-200.

17. Simon Klakegg, Jorge Goncalves, Niels van Berkel, Chu Luo, Simo Hosio and Vassilis Kostakos. 2017. Towards Commoditised Near Infrared Spectroscopy. In ACM. http://dx.doi.org/10.1145/3064663.3064738.

18. Rita Kobb, Nannette Hoffman, Robert Lodge and Sheri Kline. 2003. Enhancing elder chronic care through technology and care coordination: report from a pilot. Telemedicine Journal and eHEALTH 9, 2: 189-195.

19. Nicholas D. Lane, Mu Lin, Mashfiqui Mohammod, Xiaochao Yang, Hong Lu, Giuseppe Cardone, Shahid Ali, Afsaneh Doryab, Ethan Berke and Andrew T. Campbell. 2014. Bewell: Sensing sleep, physical activities and social interactions to promote wellbeing. Mobile Networks and Applications 19, 3: 345359.

20. Chu Luo, Angelos Fylakis, Juha Partala, Simon Klakegg, Jorge Goncalves, Kaitai Liang, Tapio Seppänen and Vassilis Kostakos. 2016. A Data Hiding Approach for Sensitive Smartphone Data. In International Joint Conference on Pervasive and Ubiquitous Computing Adjunct, ACM, 457-468. http://dx.doi.org/10.1145/2971648.2971686.

21. R. N. Madeira, Octavian Postolache, Nuno Correia and P. Silva. 2010. Designing a pervasive healthcare assistive environment for the elderly. Ubicomp.

22. Material Design for Android | Android Developers. Retrieved 22/10/2015 from http://developer.android.com/design/material/in dex.html

23. Jakob Nielsen and Rolf Molich. 1990. Heuristic Evaluation of User Interfaces. In Proceedings of the SIGCHI Conference on Human Factors in 
Computing Systems, ACM, 249-256. http://dx.doi.org/10.1145/97243.97281.

24. World Health Organization. 2013. Action on patient safety: high 5s. Retrieved 22/06/2010 from www.who.int/patientsafety/solutions/high5s/en/i ndex.html

25. Michael Prilla, Martin Degeling and Thomas Herrmann. 2012. Collaborative reflection at work: supporting informal learning at a healthcare workplace. In Proceedings of the 17th ACM international conference on Supporting group work, 55-64.

26. Parisa Rashidi and Alex Mihailidis. 2013. A survey on ambient-assisted living tools for older adults. IEEE journal of biomedical and health informatics 17, 3: 579-590.

27. Yonglin Ren, Richard Werner, Nelem Pazzi and Azzedine Boukerche. 2010. Monitoring patients via a secure and mobile healthcare system. IEEE Wireless Communications 17, 1: 59-65.

28. Centers for Medicare \& Medicaid Services. 2015. NHE fact sheet. Baltimore, Maryland. Retrieved 28/07/2015 from https://www.cms.gov/research-statistics-dataand-systems/statistics-trends-andreports/nationalhealthexpenddata/nhe-factsheet.html

29. Tarik Taleb, Dario Bottazzi, Mohsen Guizani and Hammadi Nait-Charif. 2009. ANGELAH: a framework for assisting elders at home. IEEE Journal on Selected Areas in Communications 27, 4: 480-494.

30. Monica Tentori and Jesus Favela. 2008. Monitoring behavioral patterns in hospitals through activity-aware computing. In Pervasive Computing Technologies for Healthcare, 2008. PervasiveHealth 2008. Second International Conference on, 173-176.
31. Konrad Tollmar, Frank Bentley and Cristobal Viedma. 2012. Mobile Health Mashups: Making sense of multiple streams of wellbeing and contextual data for presentation on a mobile device. In 2012 6th International Conference on Pervasive Computing Technologies for Healthcare (PervasiveHealth) and Workshops, 65-72.

32. Huey-Ming Tzeng and Shaké Ketefian. 2002. The relationship between nurses' job satisfaction and inpatient satisfaction: an exploratory study in a Taiwan teaching hospital. Journal of nursing care quality 16, 2: 39-49.

33. UN Economics and Social Affairs: World Population Prospects - Key findings \& advance tables 2015. Retrieved 28/07/2015 from http://esa.un.org/unpd/wpp/Publications/Files/K ey_Findings_WPP_2015.pdf

34. Nicole K. Valtorta, Mona Kanaan, Simon Gilbody, Sara Ronzi and Barbara Hanratty. 2016. Loneliness and social isolation as risk factors for coronary heart disease and stroke: systematic review and meta-analysis of longitudinal observational studies. Heart: heartjnl-2015-308790. http://dx.doi.org/10.1136/heartjnl-2015-308790.

35. Upkar Varshney. 2007. Pervasive healthcare and wireless health monitoring. Mobile Networks and Applications 12, 2-3: 113-127.

36. Long Wu, Jhao-Yin Li and Chu-Ying Fu. 2011. The adoption of mobile healthcare by hospital's professionals: An integrative perspective. Decision Support Systems 51, 3: 587-596. 